\title{
Role of periostin in cancer progression and metastasis: Inhibition of breast cancer progression and metastasis by anti-periostin antibody in a murine model
}

\author{
MARIKO KYUTOKU ${ }^{1}$, YOSHIAKI TANIYAMA ${ }^{1,2}$, NARUTO KATSURAGI ${ }^{3}$, HIDEO SHIMIZU $^{1,2}$, \\ YASUO KUNUGIZA ${ }^{1}$, KAZUMA IEKUSHI ${ }^{1,2}$, NOBUTAKA KOIBUCHI ${ }^{4}$, FUMIHIRO SANADA ${ }^{1}$, \\ YOSHIHIRO OSHITA $^{1}$ and RYUICHI MORISHITA ${ }^{1}$
}

\begin{abstract}
Departments of ${ }^{1}$ Clinical Gene Therapy and ${ }^{2}$ Geriatric Medicine and Nephrology, Osaka University Graduate School of Medicine, Suita, Osaka 565-0871; ${ }^{3}$ Biomedical Research Laboratories, Asubio Pharma Co., Ltd., Chuo-ku, Kobe 650-0047; ${ }^{4}$ Department of Pharmacology and Molecular Therapeutics, Kumamoto University Graduate School of Medical Sciences, Kumamoto 860-8556, Japan
\end{abstract}

Received January 13, 2011; Accepted February 23, 2011

DOI: $10.3892 /$ ijmm.2011.712

\begin{abstract}
Periostin (PN), a secreted adhesion-related protein expressed in the periosteum and periodontal ligaments, acts as a critical regulator of the formation and maintenance of bone and teeth, and also plays an important role in tumorigenesis. Although PN is highly expressed in various types of human cancers, its function is still unclear. In this study, we focused on the exon 17 region of $\mathrm{PN}$, which is alternatively spliced out. To investigate the function of full-length PN with exon 17, we produced a neutralizing antibody (PN1-Ab) against the peptide encoded by exon 17. In vivo, administration of PN1-Ab significantly inhibited the growth of primary tumors as well as metastatic tumors, associated with prevention of bone destruction, resulting in increased survival of mice. Consistent with in vivo data, the present in vitro study demonstrated that addition of full-length PN significantly inhibited cell adhesion and detached adherent cells, while PN1-Ab inhibited the action of PN in a dose-dependent manner. In addition, PN1-Ab significantly inhibited the proliferation, migration and invasion of 4T1 mouse breast cancer cells, which produced PN. Interestingly, PN1-Ab also inhibited the differentiation of osteoclasts. Overall, the present study demonstrated that PN plays a pivotal role in the progression and metastasis of breast cancer. Since administration of PN1-Ab prolonged cell survival through inhibition of the progression and metastasis of 4T1 cells, further development of the PN1-Ab such as generation of a humanized antibody may provide a new therapeutic agent against breast cancer.
\end{abstract}

Correspondence to: Professor Ryuichi Morishita, Department of Clinical Gene Therapy, Osaka University Graduate School of Medicine, 2-2 Yamada-oka, Suita, Osaka 565-0871, Japan

E-mail: morishit@cgt.med.osaka-u.ac.jp

Key words: breast cancer, periostin, anti-periostin antibody

\section{Introduction}

Periostin (PN), originally identified in the cDNA library from the mouse osteoblast cell line, MC3T3-E1, and named osteoblast-specific factor 2 (OSF-2), is a disulfide-linked $90-\mathrm{kDa}$ secretory protein $(1,2)$. Recent studies have shown that $\mathrm{PN}$ is highly expressed in various human cancers, and have suggested that $\mathrm{PN}$ promotes tumor growth and metastasis (3-5). Moreover, PN is believed to promote tumor growth and metastasis, and to be correlated with poor prognosis. Also, PN is reported to be up-regulated in the metastases of colorectal and liver cancers, suggesting its role in promoting tumor growth. Increased expression of PN has been associated with advanced carcinogenesis and cell proliferation, adhesion and migration (6-8). PN expression was readily detectable in breast tumor samples, while it was undetectable in normal breast tissue (9). In addition, serum PN levels were elevated in breast cancer patients with bone metastases (10).

Our previous study showed that overexpression of PN1 in the normal rat heart induced cardiac heart enlargement and dysfunction, while inhibition of PN1 by antisense improved cardiac dysfunction in congestive heart failure (11). PN1 was up-regulated by extension or angiotensin II in fibroblasts, and increased in myocardial infarction (12). In contrast, inhibition of PN in PN knockout mice which lacked the function of all spliced PN isoforms worsened cardiac function $(13,14)$. Importantly, the existence of various isoforms of PN is well known (15), but the functional role of PN isoforms in the progression and metastasis of cancer is still unclear $(3,16)$. In the mouse, four different isoforms from human cancer PN have been reported: full length PN, exon 17 spliced out PN, exon 21 spliced out PN and both exon 17 and exon 21 spliced out PN (17). Kim et al have shown that overexpression of spliced PN, which lacks the exon 17 region, suppresses lung metastasis of B16-F10 cells (16). This report supported the hypothesis that the exon 17 region in PN is essential to tumor progression and metastasis. Therefore, in the present study we focused on full-length PN (periostin-1, PN1), rather than 
spliced PN (periostin-2, PN2) which entails the deletion of exon 17, in breast cancer (Fig. 1A). To clarify the role of PN1, we created a neutralizing antibody (PN1-Ab) against the PN1 isoform. Here, we demonstrate that PN1 plays a crucial role in the progression and metastasis of breast cancer, suggesting that PN1-Ab might be a novel potential therapeutic drug against breast cancer.

\section{Materials and methods}

Cell culture. 4T1 cells were purchased from the American Type Culture Collection, grown in RPMI-1640 (Invitrogen) supplemented with $10 \%$ fetal bovine serum (FBS, Biowest) and 100 units/ml penicillin-streptomycin (Nacalai Tesque) under the following conditions: $5 \% \mathrm{CO}_{2}$ in air at $37^{\circ} \mathrm{C}$. Bone marrow cells were obtained from 3-day-old neonatal white rabbits as previously described (18).

RT-PCR and real-time PCR. Total RNA was prepared using Isogen (Nippon Gene) according to the manufacturer's instructions. Any DNA contamination was eliminated by DNase I treatment. First strand cDNA was synthesized according to the SuperScript First-Strand Synthesis System for RT-PCR (Invitrogen). PCR was performed using KOD plus polymerase (Toyobo) in a thermal cycler (Takara). Primer sequences were as follows: mouse PN1, forward 5'ATAACC AAAGTCGTGGAACC3' and reverse 5'TGTCTCCCTGAA GCAGTCTT3'; mouse PN2, forward 5'CCATGACTGTCTA TAGACCTG3' and reverse 5'GTCTGGCCTCTGGGTTTT3'; mouse $\beta$-tubulin, forward 5'TCACTGTGCCTGAACTTAC C3' and reverse 5'GGAACATAGCCGTAAACTGC3'; mouse glyceraldehyde-3-phosphatase dehydrogenase (GAPDH), forward 5'GGGTGGAGCCAAACGGGTC3' and reverse 5'GGAGTTGCTGTTGAAGTCGCA3'. The amplification parameters were as follows: initial denaturation at $94^{\circ} \mathrm{C}$ for 5 min followed by 40 cycles of denaturation at $94^{\circ} \mathrm{C}$ for $30 \mathrm{sec}$, annealing at $56^{\circ} \mathrm{C}$ for $30 \mathrm{sec}$ and extension at $68^{\circ} \mathrm{C}$ for $30 \mathrm{sec}$, and final extension at $72^{\circ} \mathrm{C}$ for $7 \mathrm{~min}$. Amplified PCR products were resolved on $2.0 \%$ agarose gels and visualized by ethidium bromide staining. In the same way, real-time quantitative RT-PCR was carried out using SYBR Premix Ex Taq $^{\mathrm{TM}}$ II (Takara) and an ABI PRISM ${ }^{\mathrm{TM}} 7900$ sequence detector.

Western blot analysis. Cells and tissues were lysed with RIPA buffer containing an inhibitor cocktail (Nacalai Tesque), sodium fluoride and sodium orthovanadate. After centrifugation the supernatants were subjected to 7.5\% SDS-PAGE, and electroblotted. After blocking with 5\% (w/v) skim milk (Nacalai Tesque), the membrane was incubated with PN1-Ab as the primary antibody and horseradish peroxidase-conjugated anti-rabbit IgG as the secondary antibody. Protein bands were visualized using the ECL-plus reagent (GE Healthcare). In order to raise the polyclonal antibody against exon 17 of rat PN, the peptide (TKIITKLVEPKIKVIQGSLQPIIKTE) was synthesized and coupled to KLH at Oriental Yeast Co., Ltd. The antibody was generated in immunized rabbits, and purified through an affinity column. Anti- $\alpha$-tubulin monoclonal antibody (Santa Cruz Biotechnology) was used as an internal control.
Cell adhesion assay. 4T1 cells were added to a 96-well plate coated with recombinant human PN (R\&D Systems), fibronectin (FN, Sigma), or bovine serum albumin (BSA, Sigma) at $4^{\circ} \mathrm{C}$ overnight. After incubation at $37^{\circ} \mathrm{C}$, the cells were fixed, stained with crystal violet, and measured photometrically. Moreover, to examine their ability to detach cells, PN-1, FN, or BSA were added to the 4T1 culture plate, followed by incubation, and analysis by the same procedure.

MTS and LDH assays. The MTS assay was performed with a CellTiter96 aqueous one-solution cell proliferation assay kit (Promega) according to the manufacturer's instructions. Briefly, 4T1 cells were plated in 96-well plates overnight, and treated with PN1-Ab or control IgG at different concentrations or without antibodies (only serum-free medium). Then, the MTS solution mixed with PES was directly added to the culture well and the absorbance at $490 \mathrm{~nm}$ was measured by using a microplate reader (Bio-Rad).

The LDH assay was also carried out according to the manufacturer's instructions using a CytoTox 96 kit (Promega). Briefly, 4T1 cells were seeded in 96-well plates and incubated in complete medium. The cells were then incubated in the presence of varying concentration of antibodies. LDH released in the culture supernatants with a coupled enzymatic assay was measured at the absorbance of $490 \mathrm{~nm}$.

Migration and invasion assays. Confluent cells were scraped off with a pipette tip. The wound closure was monitored and pictured. We magnified the photos and measured twelve different widths for each well and determined the average as the migration distance from the wound origin covered by cells $36 \mathrm{~h}$ after wounding.

The invasiveness of tumor cells was evaluated using the CytoSelect ${ }^{\mathrm{TM}}$ 24-well cell invasion assay (Cell Biolabs) according to the manufacturer's protocol. FBS was used as a chemoattractant.

Osteoclast differentiation. Mononuclear cells were induced to differentiate into osteoclast cells by macrophage-colony-stimulating factor (M-CSF) and receptor activator of $\mathrm{NF}-\kappa \mathrm{B}$ ligand (RANKL). Osteoclast differentiation was examined by a rat osteoclast culture system kit (Cell Garage, Tokyo, Japan). Rat osteoclast precursor cells seeded in a 24-well plate were incubated in an M-CSF (10 ng/ml) and RANKL (10 ng/ml)containing medium. PN1-Ab or control IgG were added in the culture wells of mononuclear cells with M-CSF and RANKL, and the cells were stained with tartrate-resistant acid phosphatase (TRAP) and Hoechst 33258 (Nacalai Tesque) and counted as previously described (18). Moreover, 4T1 cells were cultured in complete medium with or without antibody, and the culture supernatant was collected and used for the culture of rabbit bone marrow. The cells were stained and counted by the same procedure.

Primary and metastatic tumors in mice. Female BALB/c mice were obtained from Charles River Breeding Laboratories, and housed in the animal facility of Osaka University Medical School. Mice were inoculated with $4 \mathrm{~T} 1$ cells $\left(1 \times 10^{6}\right.$ cells) in their foot pad and administered $20 \mu \mathrm{g}$ PN1-Ab or control IgG (R\&D Systems) via a cervical vein. The tumor volume 


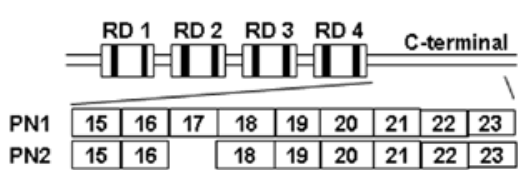

B PN1 PN2 B-tubulin

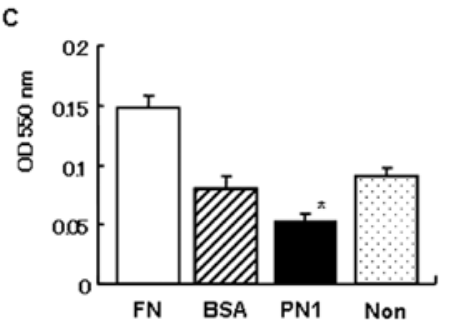

D

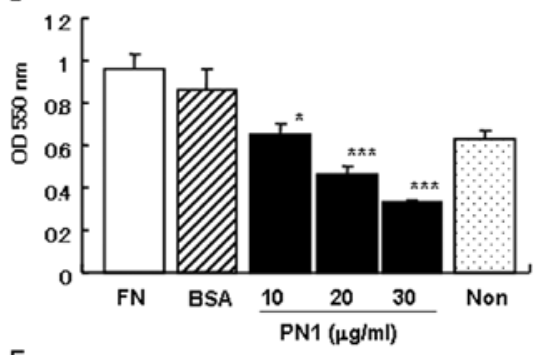

E

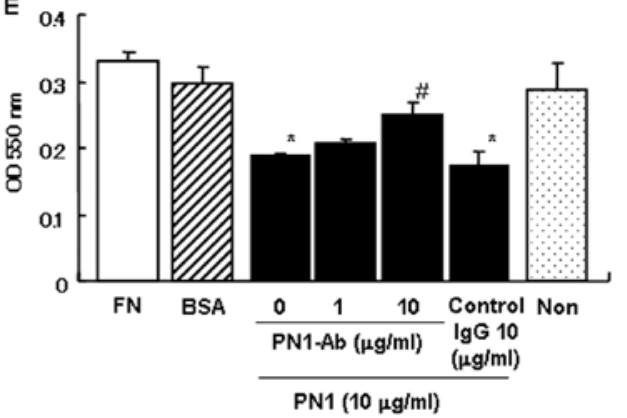

Figure 1. Periostin (PN) is an adhesion-related molecule. (A) Structure of full length PN (PN1) and spliced PN (PN2). The variants of PN are produced by alternative splicing events within the C-terminal region. Repeated domains (RDs) contain two highly conserved sequences indicated by black boxes. Exon 17 is spliced out in PN2. (B) Expression of PN mRNA in 4T1 cells was assessed by RT-PCR. (C) Reduction of 4T1 cell adhesion to the plate coated with PN1 ("P $<0.05$ vs. BSA). (D) Ability of PN to detach adherent $4 \mathrm{~T} 1$ cells $\left({ }^{*} \mathrm{P}<0.05 ;{ }^{* * * *} \mathrm{P}<0.001\right.$ vs. BSA). (E) Ability to detach adherent $4 \mathrm{~T} 1$ cells in the presence of $\mathrm{PN} 1-\mathrm{Ab}\left({ }^{*} \mathrm{P}<0.05\right.$ vs. BSA, ${ }^{*} \mathrm{P}<0.05$ vs. PN1 + control IgG, $\left.10 \mu \mathrm{g} / \mathrm{ml}\right)$. Experiments were performed in triplicate. Control IgG (normal rabbit $\left.\mathrm{IgG}\right)$ was used as an IgG control. FN, fibronectin; BSA, bovine serum albumin.

was measured every week and the number of colonies in the metastasized lung was counted 3 weeks later. Moreover, tumor tissues were scanned by CT and X-ray, and also stained with hematoxylin and eosin (H\&E) for histological analysis. All animal experiments were performed in accordance with the animal protocol procedures approved by the Animal Studies Committee of Osaka University Medical School.

In vivo lung metastasis assay. 4T1 cells $\left(1 \times 10^{4}\right.$ cells in $50 \mu 1$ PBS) were injected via a cervical vein (19). PN1-Ab (40 $\mu \mathrm{g})$ or control IgG $(40 \mu \mathrm{g})$ was given on day 7 and day 10 after cell injection, and the number of colonies formed in the lungs were counted on day 14 after cell injection.

Kaplan-Meier survival analysis. Mice were inoculated with 4T1 cells $\left(1 \times 10^{4}\right.$ cells in $100 \mu \mathrm{l}$ PBS $)$ in their foot pad and administered $40 \mu \mathrm{g}$ PN1-Ab or control IgG twice a week for 4 weeks intraperitoneally.

Statistical analysis. Data are expressed as the means \pm SEM. Statistical analysis was performed with StatView or the Student's t-test, as appropriate. P-values $<0.05$ were considered significant.

\section{Results and Discussion}

Expression of PN isoforms in breast cancer cells. We found mainly two isoforms; full-length PN (PN1) and spliced PN (PN2) in 4T1 breast cancer cells (Fig. 1A). PN1 as well as PN2 were expressed in malignant 4T1 (Fig. 1B). As the function of the PN isoforms has not yet been clarified in cancer cells, we first examined the role of PN1 in cell adhesion, since addition of PN1 stimulated cell detachment in fibroblasts. Interestingly, coating with PN1 significantly inhibited cell adhesion of 4T1 cells to the dishes as compared to BSA, the control protein (Fig. 1C). Moreover, addition of PN1 resulted in a significant increase in the detachment of adherent 4T1 cells in a dosedependent manner, while the addition of FN or BSA did not affect it (Fig. 1D). These results were consistent with the data from fibroblasts (11). We confirmed that PN is an adhesionrelated molecule. Thus, we created PN1-Ab against exon 17, since PN1 might play an important role in the metastasis of cancer. Expectedly, addition of PN1-Ab exhibited neutralizing activity against PN1. As shown in Fig. 1E, addition of PN1-Ab significantly inhibited PN1-induced cell detachment in a dosedependent manner.

PN1-Ab inhibits cell proliferation, induces tumor necrosis and blocks cell migration and invasion in vitro. In addition to the inhibition of cell detachment by PN1, PN1-Ab at a concentration of $40 \mu \mathrm{g} / \mathrm{ml}$ decreased the proliferation of $4 \mathrm{~T} 1$ cells, as demonstrated using the MTS assay (Fig. 2A) and increased cytotoxicity, as shown with the LDH assay (Fig. 2B). As 4T1 cells are highly migratory and invasive in vitro, which is a trait reflected in the metastasis observed in vivo, we next examined whether PN1-Ab affected cell motility and invasiveness using a scratch wound healing assay and a Boyden chamber assay, respectively. Addition of $\mathrm{PN} 1-\mathrm{Ab}$ significantly reduced cell migration at both 20 and $50 \mu \mathrm{g} / \mathrm{ml}$ (Fig. 2C), and invasion into the Matrigel at $40 \mu \mathrm{g} / \mathrm{ml}$ (Fig. 2D), as compared to the control IgG group. Although it is unclear whether the previous anti-proliferative activity and cytotoxicity of PN1-Ab at high concentrations affect the migration and invasion, these results indicate that PN1-Ab can attenuate the malignant activity of 4T1 cells in vitro. We therefore, selected 4T1 cells to study the role of PN1 in tumor progression and metastasis, since animal 
A

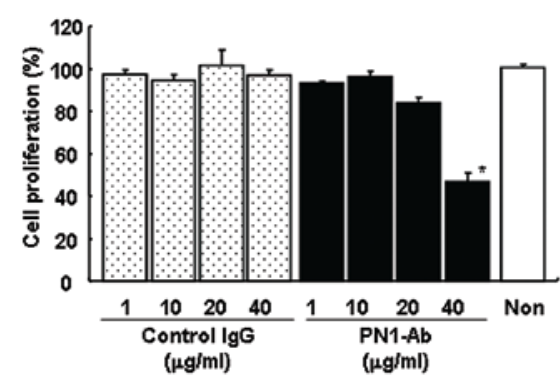

C

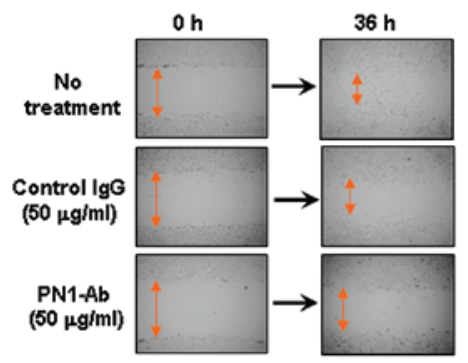

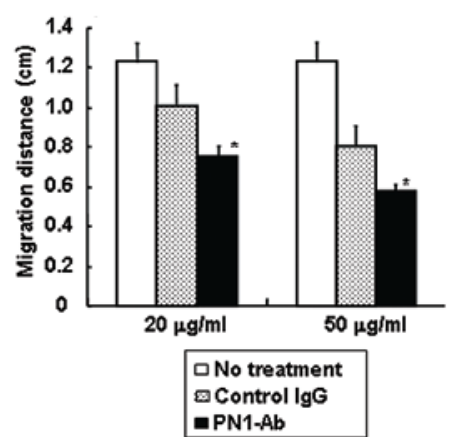
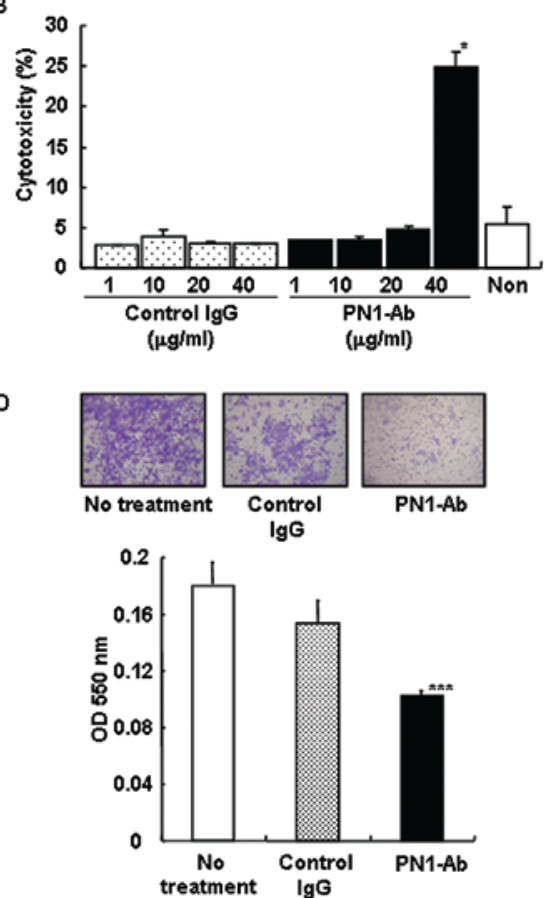

Figure 2. The anti-periostin antibody (PN1-Ab) inhibits the malignant activity of 4T1 cells in vitro. (A) MTS assay. The number of viable cells was measured ("P<0.05 vs. control IgG $40 \mu \mathrm{g} / \mathrm{ml}$ ). (B) LDH assay. Necrotic cells were measured ("P $<0.05$ vs. control IgG $40 \mu \mathrm{g} / \mathrm{ml}$ ). (C) Scratch wound healing assay. Migration of 4T1 cells with addition of PN1-Ab ("P<0.05 vs. control IgG 20 or $50 \mu \mathrm{g} / \mathrm{ml}$ ). (D) Boyden chamber assay. Invasion of $4 \mathrm{~T} 1$ cells in Matrigel was quantified $\left({ }^{* * *} \mathrm{P}<0.001\right.$ vs. control $\left.\operatorname{IgG} 40 \mu \mathrm{g} / \mathrm{ml}\right)$. Experiments were performed in triplicates. Control $\operatorname{IgG}$ was used as the control.

A
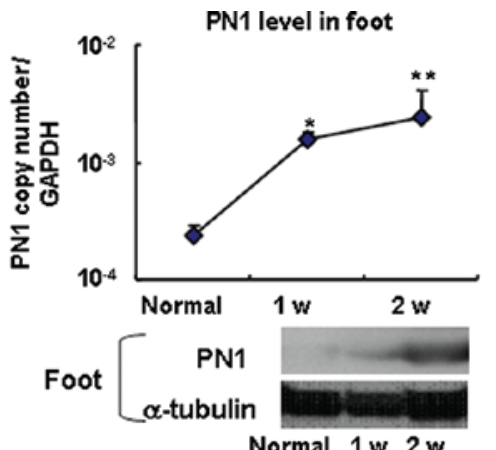

Normal 1 w 2 w

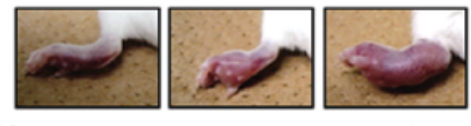

time
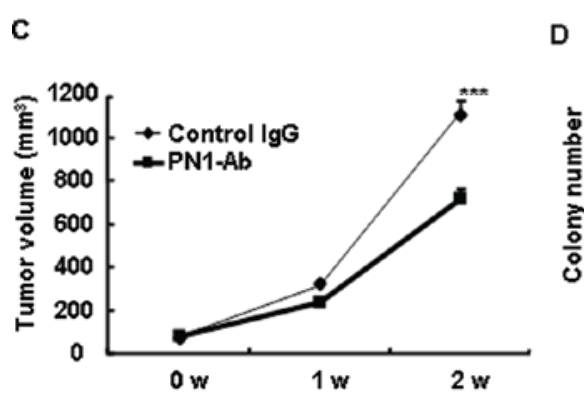

B
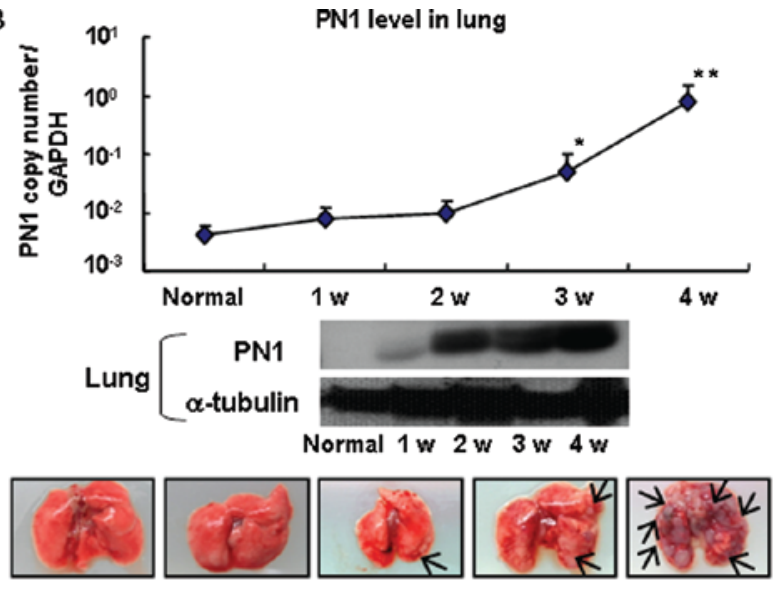

time

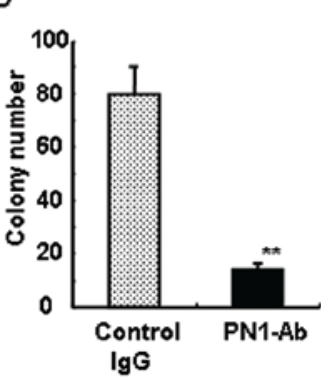

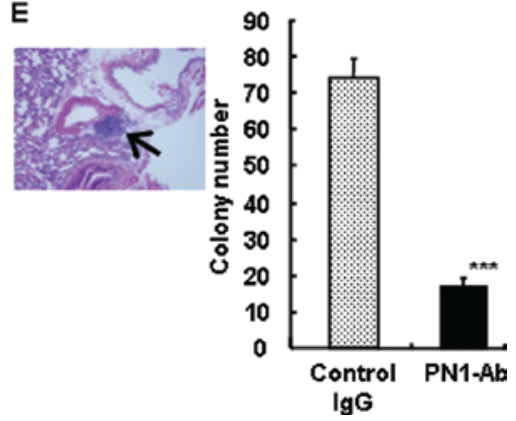

Figure 3. Periostin (PN) expression increases with tumor growth, and PN1-Ab suppresses tumor growth in primary and metastatic tissue evaluated up to 4 weeks (w) after treatment. (A and B) mRNA and protein expression levels of PN1 in lung metastasis model were analyzed by real-time PCR (primary, n=3; metastatic, $n=12 ;{ }^{*} \mathrm{P}<0.05 ;{ }^{* *} \mathrm{P}<0.01$ vs. normal) and Western blot analysis ( $\mathrm{n}=3$ each). (A) Primary tumors in the foot. (B) Metastatic tumors in the lung. (C and D) PN1-Ab administration in a lung metastasis mouse model. (C) Primary tumor volume ( $\mathrm{n}=10$ each, ${ }^{* * *} \mathrm{P}<0.001$ vs. control IgG). (D) The number of colonies in the lung ( $\mathrm{n}=9$ each, ${ }^{* * * *} \mathrm{P}<0.001$ vs. control $\left.\mathrm{IgG}\right)$. (E) PN1-Ab administration in the in vivo metastasis mouse model $\left(\mathrm{n}=8\right.$ each, $\left.{ }^{* * * *} \mathrm{P}<0.001\right)$. The black arrow denotes $4 \mathrm{~T} 1$ cells, which show a much higher nuclear/cytoplasmic ratio than other areas. $4 \mathrm{~T} 1$ cells were identified by $\mathrm{H} \& \mathrm{E}$ staining $(\mathrm{n}=3)$. Control IgG was used as a control. 

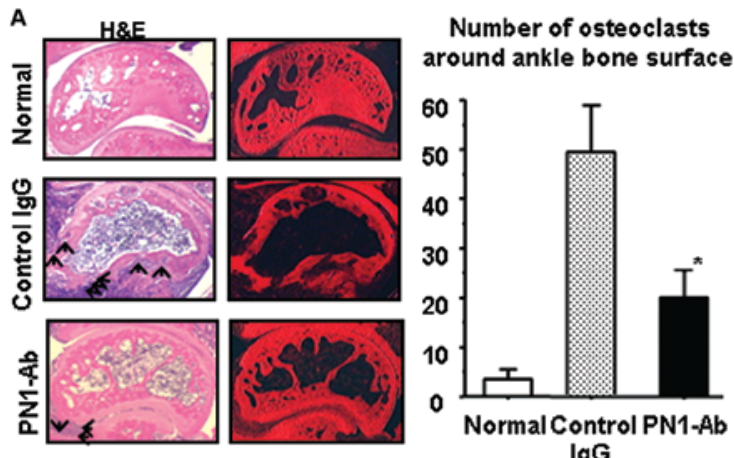

$\lg \mathrm{G}$

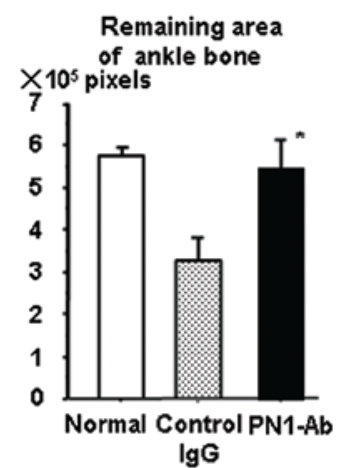

D

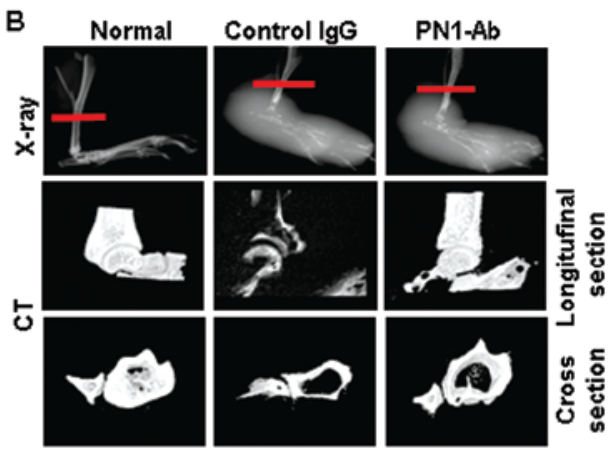

E
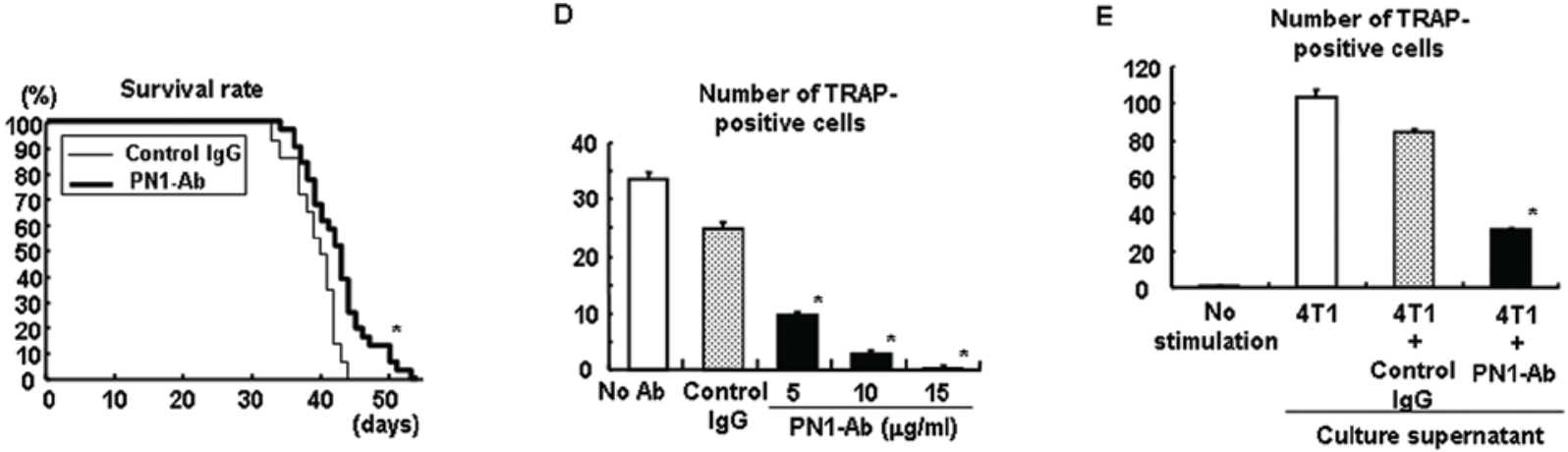

Figure 4. PN1-Ab suppresses bone invasion and increases mouse survival rate. (A) The remaining area of the bone and the number of osteoclasts around the ankle bone ( $\mathrm{n}=5$ each, ${ }^{\mathrm{P}}<0.05$ vs. control IgG). Black arrows denote multinucleated osteoclasts around the bone surface. (B) X-ray and $\mathrm{CT}$ scanning in the primary foot ( $\mathrm{n}=3$ each). (C) Kaplan Meier survival analysis $\left(\mathrm{n}=29\right.$, control IgG; $n=31$, PN1-Ab; ${ }^{*} \mathrm{P}<0.05$ vs. control IgG). (D and E) The number of osteoclasts. (D) Direct differentiation of osteoclasts by M-CSF and RANKL ( $\mathrm{P}<0.05$ vs. control IgG $15 \mu \mathrm{g} / \mathrm{ml})$. (E) Indirect osteoclast differentiation ( $\mathrm{P}<0.05 \mathrm{vs.} \mathrm{control}$ $\mathrm{IgG} 15 \mu \mathrm{g} / \mathrm{ml})$. Experiments were performed in triplicate. Control IgG was used as the control.

models using 4T1 cells can be used to study all steps of tumor progression and metastasis in the lung (20).

PN1-Ab inhibits tumor growth, metastasis and bone destruction in vivo. Given the inhibitory effects of PN1-Ab on cancer cells, we administered PN1-Ab to mice of a lung metastasis model, in which 4T1 cells were initially inoculated into the mouse foot pad, to investigate the effects of $\mathrm{PN} 1-\mathrm{Ab}$ in vivo. In this model, metastasis to the lung from the foot pad occurred two to three weeks after cell inoculation, while necrosis of primary tumors started two or three weeks after cell inoculation. First, we analyzed PN1 expression at the transcription and protein level at various time intervals. The level of PN1 mRNA in inoculated footpads increased up to 10-fold as compared to that in normal footpads, whereas PN1 mRNA markedly increased up to 100-fold in the lung as compared to that in the normal lung (Fig. 3A and B). On the other hand, the protein expression level of PN1 rose steadily as the tumors grew. In contrast, the expression of PN1 was not detectable in normal feet and lungs by Western blot analysis. The difference in the expression was reflected in the tumor progression and in the timing of the metastasis to the lung from the foot. This marked increase in PN1 implies a role of PN1 in tumor progression and detachment from the primary tumor. It was thought that PN1 expression in tumor tissues including tumor cells increased along with tumor growth. Thus, we administered PN1-Ab to examine the changes in primary tumor volume and the number of lung-metastasized colonies. Importantly, a single injection of PN1-Ab signifi- cantly inhibited primary tumor growth after two weeks in the footpad (Fig. 3C), and decreased the number of metastatic colonies in the lung after three weeks as compared to the control IgG group (Fig. 3D). Thus, PN1-Ab may have two beneficial effects: i) the decrease in metastasis from the reduction of primary tumor and ii) suppression of tumor growth in the metastatic site. To confirm the inhibitory effects of $\mathrm{PN} 1-\mathrm{Ab}$ on metastasis, we further employed an experimental lung metastasis model. In this model, cells were inoculated into a cervical vein, reached the lung, and had grown at 4 days after inoculation (Fig. 3E, H\&E staining). PN1-Ab was administrated twice, on days 7 and 10 after inoculation, which significantly decreased the number of colonies on day 14 (Fig. 3E). Indeed, the number of colonies was significantly decreased, suggesting that PN1 might be important for colony growth in the lung. PN1-Ab could inhibit tumor growth in both primary and metastatic tissue.

PN1-Ab suppressed bone destruction in vivo and osteoclast differentiation in vitro. In addition, invasive destruction involving the ankle bones in the former foot pad model was markedly inhibited by PN1-Ab, accompanied by a significant decrease in the number of osteoclasts in the vicinity (Fig. 4A). Multinucleated cells adjacent to the bone, where inflammation occurred on its surface, were counted as osteoclasts. Radiography and CT scan demonstrated that PN1-Ab significantly attenuated the decrease in bone mass, as compared to the control IgG group (Fig. 4B). The control IgG group exhibited severe bone destruction, particularly in the 
bone marrow cavity, both in longitudinal and cross sections, while the PN1-Ab group showed inhibition of osteolysis by 4T1 cells. Most importantly, treatment with PN1-Ab resulted in a significant increase in the survival rate (Fig. 4C).

Finally, we explored the relationship between bone and PN1, since PN originates in osteoblasts $(1,2)$. M-CSF and RANKL are necessary for the maturation of osteoclasts from osteoclast precursors, and mature (active) osteoclasts can be distinguished by assessing TRAP activity. We first evaluated the differentiation of osteoclasts using PN1-Ab. In a culture of osteoclast precursor cells with M-CSF and RANKL, addition of PN1-Ab significantly inhibited osteoclast maturation, decreasing TRAP-positive cells in a dose-dependent manner (Fig. 4D). Moreover, in a bone marrow culture, the culture supernatant of $4 \mathrm{~T} 1$ cells induced differentiation to mature osteoclasts, which was decreased by the culture supernatant of 4T1 cells with PN1-Ab at $20 \mu \mathrm{g} / \mathrm{ml}$ (Fig. 4E). The results indicate that $4 \mathrm{~T} 1$ cells secreted $\mathrm{PN}-1$, which is involved in osteoclast differentiation. Overall, our present data demonstrate that PN1-Ab significantly improves survival in a mouse cancer model, through inhibition of primary tumor growth and of metastasis. Further development of PN1-Ab as a humanized antibody may provide a new therapeutic agent against breast cancer.

\section{Acknowledgements}

This study was partially supported by a Grant-in-Aid from Asubio Pharma Co., Ltd., the Japan Science and Technology Agency, and Research Fellowships from the Japan Society for the Promotion of Science for Young Scientists. The authors thank all the members of the Department of Clinical Gene Therapy for their advice.

\section{References}

1. Takeshita S, Kikuno R, Tezuka K and Amann E: Osteoblastspecific factor 2: cloning of a putative bone adhesion protein with homology with the insect protein fasciclin I. Biochem J 294: 271-278, 1993.

2. Horiuchi K, Amizuka N, Takeshita S, et al: Identification and characterization of a novel protein, periostin, with restricted expression to periosteum and periodontal ligament and increased expression by transforming growth factor beta. J Bone Miner Res 14: 1239-1249, 1999.

3. Bao S, Ouyang G, Bai X, et al: Periostin potently promotes metastatic growth of colon cancer by augmenting cell survival via the Akt/PKB pathway. Cancer Cell 5: 329-339, 2004.
4. Tai IT, Dai M and Chen LB: Periostin induction in tumor cell line explants and inhibition of in vitro cell growth by antiperiostin antibodies. Carcinogenesis 26: 908-915, 2005.

5. Puglisi F, Puppin C, Pegolo E, et al: Expression of periostin in human breast cancer. J Clin Pathol 61: 494-498, 2008.

6. Siriwardena BS, Kudo Y, Ogawa I, et al: Periostin is frequently overexpressed and enhances invasion and angiogenesis in oral cancer. Br J Cancer 95: 1396-1403, 2006.

7. Erkan M, Kleeff J, Gorbachevski A, et al: Periostin creates a tumor-supportive microenvironment in the pancreas by sustaining fibrogenic stellate cell activity. Gastroenterology 132: 1447-1464, 2007.

8. Kudo Y, Ogawa I, Kitajima S, et al: Periostin promotes invasion and anchorage-independent growth in the metastatic process of head and neck cancer. Cancer Res 66: 6928-6935, 2006.

9. Shao R, Bao S, Bai X, et al: Acquired expression of periostin by human breast cancers promotes tumor angiogenesis through up-regulation of vascular endothelial growth factor receptor 2 expression. Mol Cell Biol 24: 3992-4003, 2004.

10. Sasaki H, Yu CY, Dai M, et al: Elevated serum periostin levels in patients with bone metastases from breast but not lung cancer. Breast Cancer Res Treat 77: 245-252, 2003.

11. Katsuragi N, Morishita R, Nakamura N, et al: Periostin as a novel factor responsible for ventricular dilation. Circulation 110: 1806-1813, 2004.

12. Iekushi K, Taniyama Y, Azuma J, et al: Novel mechanisms of valsartan on the treatment of acute myocardial infarction through inhibition of the antiadhesion molecule periostin. Hypertension 49: 1409-1414, 2007.

13. Norris RA, Moreno-Rodriguez RA, Sugi Y, et al: Periostin regulates atrioventricular valve maturation. Dev Biol 316: 200-213, 2008.

14. Shimazaki M, Nakamura K, Kii I, et al: Periostin is essential for cardiac healing after acute myocardial infarction. J Exp Med 205: 295-303, 2008.

15. Kim CJ, Isono T, Tambe Y, et al: Role of alternative splicing of periostin in human bladder carcinogenesis. Int $\mathrm{J}$ Oncol 32: 161-169, 2008.

16. Kim CJ, Yoshioka N, Tambe Y, Kushima R, Okada Y and Inoue $\mathrm{H}$ : Periostin is down-regulated in high grade human bladder cancers and suppresses in vitro cell invasiveness and in vivo metastasis of cancer cells. Int J Cancer 117: 51-58, 2005.

17. Shimazaki M and Kudo A: Impaired capsule formation of tumors in periostin-null mice. Biochem Biophys Res Commun 367: 736-742, 2008.

18. Shimizu H, Sakamoto M and Sakamoto S: Bone resorption by isolated osteoclasts in living versus devitalized bone: differences in mode and extent and the effects of human recombinant tissue inhibitor of metalloproteinases. J Bone Miner Res 5: 411-418, 1990.

19. Holden S, Lan Y, Pardo A, Wesolowski J and Gillies S: Augmentation of antitumor activity of an antibody-interleukin 2 immunocytokine with chemotherapeutic agents. Clin Cancer Res 7: 2862, 2001

20. Yang J, Mani SA, Donaher JL, et al: Twist, a master regulator of morphogenesis, plays an essential role in tumor metastasis. Cell 117: $927-939,2004$. 